\title{
A novel de novo KCNQ2 mutation in a child with treatment- resistant early-onset epileptic encephalopathy
}

\author{
Christina Benetou ${ }^{1}$, Stavroula Papailiou ${ }^{1}$, Despoina Maritsi ${ }^{1}$, Katherine Anagnostopoulou ${ }^{2}$, \\ Harry Kontos ${ }^{2}$, Georgios Vartzelis ${ }^{1}$ \\ ${ }^{12 n d}$ Department of Pediatrics, 'P\&A Kyriakou' Children's Hospital, Athens University, Athens; ${ }^{2}$ Department of Molecular \\ Genetics, Genomedica S.A., Piraeus, Greece. E-mail: kat_anag@yahoo.com \\ Received: 28th November 2017, Revised: 17th May 2018, Accepted: 21st May 2018
}

\begin{abstract}
SUMMARY: Benetou C, Papailiou S, Maritsi D, Anagnostopoulou K, Kontos H, Vartzelis G. A novel de novo KCNQ2 mutation in a child with treatmentresistant early-onset epileptic encephalopathy. Turk J Pediatr 2019; 61: 279-281.

Mutations in KCNQ2 gene, encoding for voltage-gated $\mathrm{K}^{+}$channel subunit, may result in a wide spectrum of early-onset epileptic disorders. The phenotype of the disease varies from "benign familial neonatal seizures" to "severe epileptic encephalopathies". In this report, we present a novel mutation [namely: c.683A $>\mathrm{G}$ (p.His228Arg)], as a presumable cause of severe infantile-onset neonatal seizures, in a 3-month old boy. The seizures have been poorly responsive to various pharmacological treatments, with phenytoin and carbamazepine presenting with the most favourable results so far. The study of our patient could help to further clarify the clinical manifestations of KCNQ2 mutations, revealing a previously unreported mutation.
\end{abstract}

Key words: drug-resistant epilepsy, KCNQ2 mutation, early-onset epileptic encephalopathy.

The KCNQ2 gene encodes for voltage-gated $\mathrm{K}^{+}$ channel subunits, which produce the neuronal M-current, a potassium current which acts by inhibiting neuronal excitation. The mechanism, through which this inhibition is mediated, has been identified by various studies as the regulation of a neuron's ability to produce an action potential. ${ }^{1}$ To date, it has been suggested that even a partial loss of function of these potassium channels, due to various mutations of the genes, can lead to the expression of an epileptic phenotype, ranging from "benign neonatal seizures" (BFNS) to "severe epileptic encephalopathies". ${ }^{2}$

Since the initial description of epileptic diseases in neonates due to mutations in the KCNQ2 channel by Wang et $\mathrm{al}^{3}$. in 1998, significant progress has been made regarding our knowledge about the specific clinical characteristics and genetic mechanisms relevant to this wide spectrum of disorders. In fact, it has been shown that BFNS are usually of familial origin, showing autosomal dominant inheritance, as opposed to epileptic encephalopathies, which tend to arise most frequently de novo. ${ }^{4}$ Here, a Greek patient with epileptic encephalopathy is reported, carrying a novel de novo mutation of the KCNQ2 gene.

\section{Case Report}

The infant was admitted to our department on day 25 of life due to seizures, which appeared on the $3^{\text {rd }}$ day of life. He was the second child of two unrelated caucasian parents and perinatal history was uneventful. Seizure semiology consisted of tonic spasms of the upper limbs, opisthotonus, abduction and adduction of the arms and gaze fixation with postictal intense crying. The episodes gradually deteriorated and at the time of the admission he suffered numerous episodes during the day, each one lasting approximately 20 to 30 seconds. The neurological examination revealed no other specific pathological signs, apart from intermittent back arching and irritability. He underwent extensive diagnostic work-up, including metabolic screening, analysis of cerebrospinal fluid for glucose, neurotransmitters and amino acids and a brain 


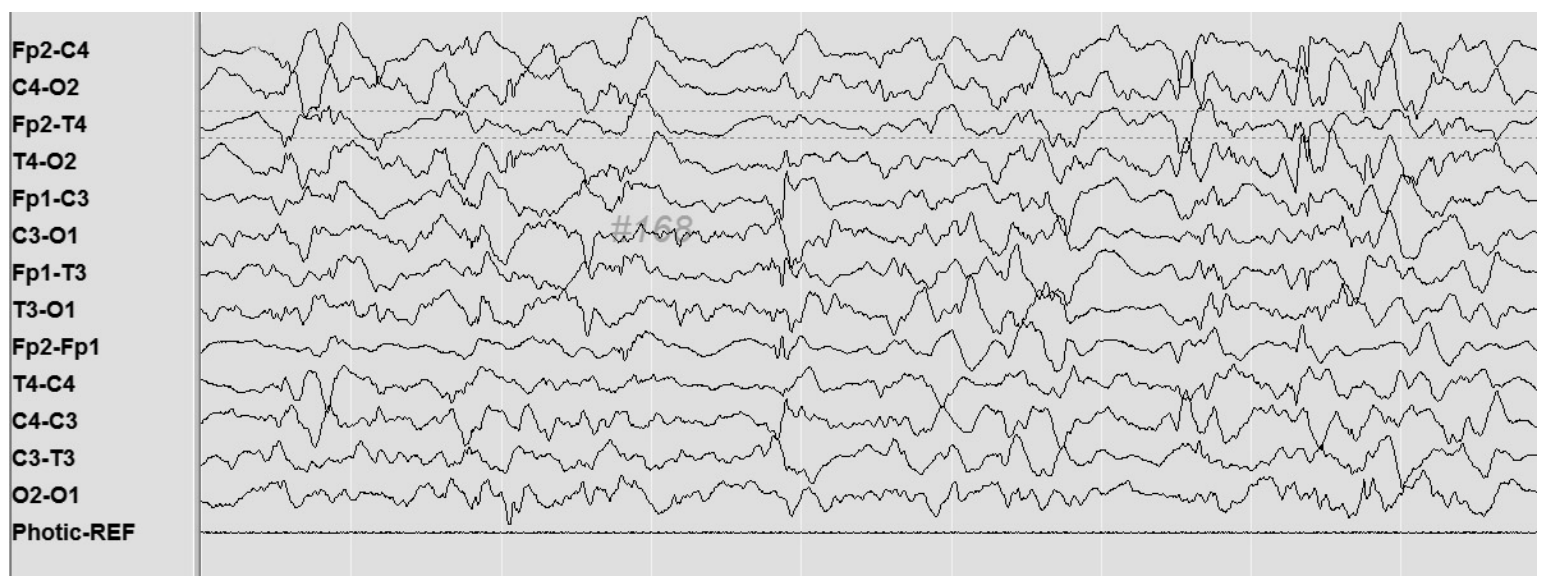

Fig. 1. Electroencephalogram of the patient demonstrating mild discontinuity and multifocal epileptic spikes.

magnetic resonance imagining (MRI), all of which were normal. An electroencephalogram (EEG) revealed multifocal epileptic spikes of low amplitude with mild discontinuation (Fig. 1).

The therapeutic approach of this patient has been challenging, since his epilepsy proved drug resistant. He was tried on several anticonvulsant medications, namely levetiracetam, pyridoxine, pyridoxal 5' phospate, phenobarbital and clobazam with no obvious improvement. However, the frequency of the episodes showed significant reduction with the administration of phenytoin, in the context of a multidrug regimen. Later in the course of the disease, oxcarbazepine was also administered, resulting in further improvement of the patient's condition. Ketogenic diet was also administered and withdrawn, having shown no beneficial impact to the overall outcome for the patient.

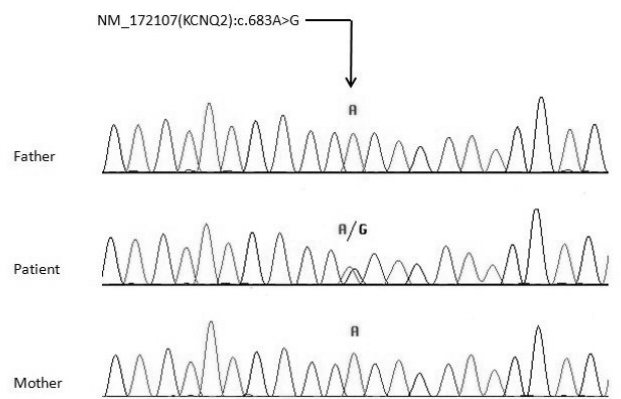

Fig. 2. Confirmation of the KCNQ2 mutation (c.683A $>\mathrm{G}$, p.His228Arg) by Sanger sequencing in patient's peripheral blood. The mutation was heterozygous and de novo. Peripheral blood of both parents was negative for the KCNQ2 mutation.
Informed consent was received from the family and DNA analysis was carried out by means of whole exome sequencing according to standard procedures. Briefly, exome capture was performed using the SureSelect V5+UTR post technology (Agilent Technologies, Santa Clara, CA, USA) and the sequencing was performed in an Illumina HiSeq 2000 sequencer (100 bp paired-end, Illumina, San Diego, CA, USA). Read mapping, variant calling and variant annotation were performed using the softwares BWA-0.7.12, GATKv3.4.0 and SnpEff_v4.1g, respectively. Variant analysis was focused on genes known to cause seizures and/or earlyonset epileptic encephalopathies.

A single heterozygous missense mutation of KCNQ2 (c.683A>G, p.His228Arg) was identified. The mutation was confirmed by bidirectional Sanger sequencing on an ABI 3500xL (Life Technologies, Carlsbad, CA). Subsequent Sanger sequencing of parental samples showed that both were negative for the mutation leading to the assumption that the mutation was likely de novo, providing strong evidence of pathogenicity (Fig. 2). The variant was absent in control population databases including ExAC (http:// exac.broadinstitute.org/) and 1000 Genomes (http://browser.1000genomes.org/index. html). Furthermore, the amino acid change involves a highly conserved residue, while changes observed in neighbouring amino acids are pathogenic or likely pathogenic. More importantly, a known pathogenic mutation has been previously reported ${ }^{5}$, involving the same codon (p.His228Gln, rs118192204). Although 
functional studies for the identified mutation have not been performed, it seems that this region and this residue in particular may be of high functional importance.

\section{Discussion}

There is a range of phenotypes linked to genetic KCNQ2 mutations. Specifically, BFNS commonly present as recurring seizures that appear during the first days of life. With an interval of weeks to months, the seizures subside, leaving no residues concerning psychomotor development. ${ }^{6}$ Interestingly enough, the interictal EEG appears mostly normal, as do all neuroradiological examinations. ${ }^{2}$ On the other hand, de novo mutations of the KCNQ2 gene are linked to a more alarming clinical presentation, with treatment-resistant seizures of higher frequency, which are features of an early-onset epileptic encephalopathy. ${ }^{7}$ These patients often demonstrate multifocal epileptic spikes or a suppression-bust pattern in the interictal EEG. ${ }^{8}$ The condition of early-onset epileptic encephalopathy has also been linked to developmental delay, as opposed to BFNS. Notably, KCNQ2 mutations are among the most common causes of Ohtahara syndrome, a progressive epileptic encephalopathy of neonatalonset, whose main characteristics are intractable episodes of tonic spasms accompanied by partial seizures. ${ }^{4}$ This syndrome also involves severe retardation, both mental and physical. ${ }^{9}$

Through electrophysiological studies and computational modelling, it has been suggested that the epileptic phenotype can arise both by gain-of-function mutations of the KCNQ2 gene, that stabilize the activated state of the channel, and by loss-of-function mutations, that decrease conductance of the M-current. ${ }^{10,11}$ Various pathophysiological pathways have been proposed, aiming to explain this increase in neuronal excitability, caused by both types of alterations to the gene. ${ }^{5}$

In this case we present a previously unreported mutation of the gene aiming to further expand our knowledge regarding these challenging cases. We believe that by continuously deepening our comprehension of the disease, novel treatment options and diagnostic modalities will arise.

\section{REFERENCES}

1. Miceli F, Vargas E, Bezanilla F, Taglialatela M. Gating currents from Kv7 channels carrying neuronal hyperexcitability mutations in the voltage-sensing domain. Biophys J 2012; 102: 1372-1382.

2. Borgatti R, Zucca C, Cavallini A, et al. A novel mutation in KCNQ2 associated with BFNC, drug resistant epilepsy, and mental retardation. Neurology 2004; 63: 57-65.

3. Wang HS, Pan Z, Shi W, et al. KCNQ2 and KCNQ3 potassium channel subunits: molecular correlates of the M-channel. Science 1998; 282: 1890-1893.

4. Miceli F, Soldovieri MV, Ambrosino P, et al. Early-onset epileptic encephalopathy caused by gain-of-function mutations in the voltage sensor of Kv7.2 and Kv7.3 potassium channel subunits. J Neurosci 2015; 35: 3782-3793.

5. Singh NA, Westenskow P, Charlier C, et al; BFNC Physician Consortium. KCNQ2 and KCNQ3 potassium channel genes in benign familial neonatal convulsions: expansion of the functional and mutation spectrum. Brain 2003; 126(Pt 12): 2726-2737.

6. Miraglia del Giudice E, Coppola G, Scuccimarra G, Cirillo G, Bellini G, Pascotto A. Benign familial neonatal convulsions (BFNC) resulting from mutation of the KCNQ2 voltage sensor. Eur J Hum Genet 2000; 8: 994-997.

7. Numis AL, Angriman M, Sullivan JE, et al. KCNQ2 encephalopathy: delineation of the electroclinical phenotype and treatment response. Neurology 2014; 82: 368-370.

8. Milh M, Boutry-Kryza N, Sutera-Sardo J, et al. Similar early characteristics but variable neurological outcome of patients with a de novo mutation of KCNQ2. Orphanet J Rare Dis 2013, 8: 80.

9. Gürsoy S, Erçal D. Diagnostic approach to genetic causes of early-onset epileptic encephalopathy. J Child Neurol 2016; 31: 523-532.

10. Milh M, Cacciagli P, Ravix C, et al. Severe neonatal seizures: From molecular diagnosis to precision therapy? Rev Neurol (Paris) 2016; 172: 171-173.

11. Battefeld A, Tran BT, Gavrilis J, Cooper EC, Kole MH. Heteromeric Kv7.2/7.3 channels differentially regulate action potential initiation and conduction in neocortical myelinated axons. J Neurosci 2014; 34: 3719-3732. 